\title{
PILL-ID: Matching and Retrieval of Drug Pill Imprint Images
}

\author{
Young-Beom Lee \\ Dept. of Brain and Cognitive Engineering \\ Korea University \\ Seoul, Korea \\ young-beom_lee@korea.ac.kr
}

\author{
Unsang Park and Anil K. Jain \\ Dept. of Computer Science \& Engineering \\ Michigan State University \\ E. Lansing, MI, USA 48824 \\ \{parkunsa,jain\}@cse.msu.edu
}

\begin{abstract}
Automatic illicit drug pill matching and retrieval is becoming an important problem due to an increase in the number of tablet type illicit drugs being circulated in our society. We propose an automatic method to match drug pill images based on the imprints appearing on the tablet. This will help identify the source and manufacturer of the illicit drugs. The feature vector extracted from tablet images is based on edge localization and invariant moments. Instead of storing a single template for each pill type, we generate multiple templates during the edge detection process. This circumvents the difficulties during matching due to variations in illumination and viewpoint. Experimental results using a set of real drug pill images (822 illicit drug pill images and 1,294 legal drug pill images) showed $76.74 \%$ (93.02\%) rankone (rank-20) matching accuracy.
\end{abstract}

Keywords-Pattern recognition systems and applications; Multimedia analysis, indexing, and retrieval

\section{INTRODUCTION}

Illicit drugs, widely circulated in the international market, are one of the major factors influencing criminal activity. They also lead to additional enforcement and tracking expense for law enforcement units. More than 35 million individuals either used illicit or abused prescription drugs in the United States alone in 2007. The U.S. federal government has allocated more than $\$ 14$ billion for drug treatment and prevention, counterdrug law enforcement, drug interdiction and international counterdrug assistance in 2009 [1]. Drug trafficking is also one of the major factors behind violent and other illegal activities [2].

U.S. Drug Enforcement Administration's Office of Forensic Sciences (DEA) has been publishing Microgram Bulletin and Microgram Journal to assist and serve forensic scientists for detection and analyses of drug-related substances [3]. Food and Drug Administration (FDA) regulation [4] requires that every prescription pill or capsule sold in the market must have unique-look for easy identification in terms of size, shape, color and imprint. Among those features, imprint is an indented or printed mark on a pill, tablet or capsule. Imprints can be a symbol, text, set of digits, or any combination of them [5]. Drug makers use imprints to identify the chemical substance and their quantity in each pill and put special imprint on the pill for advertisement purposes. When a new illicit psychoactive substance is first detected in the

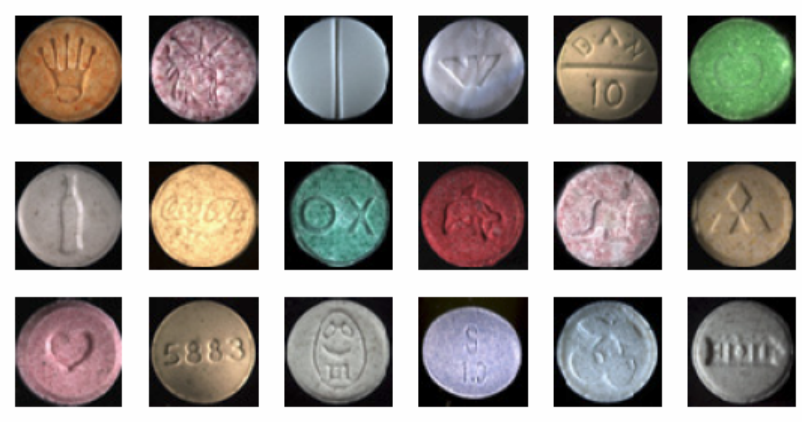

Figure 1. Example images of illicit drug pills or tablets.

market, its information is recorded in the law enforcement databases [6]. This information includes chemical and physical description, where the physical description includes size, color, imprints, etc. Fig. 1 shows example images of illicit drug pills.

Law enforcement units would like to automatically extract the information about the drug pill (i.e., type of pill, manufacturing location and the manufacturer) from the imprints by matching them with known patterns in their databases. Unlike legal drug pills that can be identified from the information provided by pharmaceutical companies and enrolled in FDA database, matching imprints with the patterns of previously seized pills is the most effective way to identify illicit drug pills. Therefore, it is important to develop an image based matching tool to automatically identify illicit drug pills based on their imprint, size, shape, color, etc. There are a few web sites that provide keyword-based legal drug pill identification tools [5] [7]. The keywords are based on the size, shape and color of the pill (e.g., round, diamond, red, etc.), but do not utilize the imprint.

There have been only a few studies on automatic pill identification. Geradts et al. [8] used aspect ratio, circularity, moment invariants and gradients. Only 75 duplicate images taken from three different pills were used for the image retrieval experiment out of a drug tablet database containing 432 images.

To develop a successful automatic pill matching system, 
it is important to generalize the variations in the appearance of the pills, due for instance to changes in viewpoint, illumination or occlusion [9]. We utilize the edge information to characterize the imprint patterns on the drug pill images. Edge detection is one of the most useful image processing methods to extract object features. It serves to simplify image analysis by drastically reducing the amount of data to be processed, while at the same time preserving useful structural information about object shape and boundary [10]. Given the processed edge image, invariant moment features proposed by $\mathrm{Hu}$ [11] and grid intensity methods are used to generate feature vectors from the edge image. With the extracted feature vectors, we use the L2-norm to compute the similarity between two pill images. The proposed method is evaluated on a database with 822 illicit drug pill images and 1,294 legal drug pill images. To the best of our knowledge, this is the first effort in developing an automatic pill identification based on imprints using a a large scale real drug pill image database.

\section{FEATURE EXTRACTION AND MATCHING}

Since the imprint on the pill is only characterized by shape and not by texture, we use one of the most popular edge detection methods, Canny edge detector [10], to localize the imprint shape and pill boundary. Feature vector is generated based on edge values using invariant moments and the grid structure. The overall process is shown in Fig. 2 .

\section{A. Preprocessing}

All the drug pills in our database were already imaged against a uniform background. This simplifies the segmentation process to tightly crop the pill image. The cropped image is normalized into a preset size of $200 \times 200$ pixels.

\section{B. Edge detection}

Canny edge detection method consists of six steps: (i) Gaussian smoothing, (ii) gradient computation with Sobel operator [12], (iii) calculating edge orientation, (iv) quantifying edge orientation, (v) non-maximum suppression and (vi) thresholding with hysteresis. We did not use the edge orientation (steps (iii), (iv) and (v)) to save computation time.

After applying the Sobel operator, the outermost distinctive boundary, which is circular for most of the pill images in our database, is removed. Next, we perform binarization using thresholding with hysteresis operation. Since, the extracted edges depend on the imaging condition of the input pill, such as the variations in view point and illumination, we applied a set of threshold values to obtain multiple edge images for each pill as described in the next section.

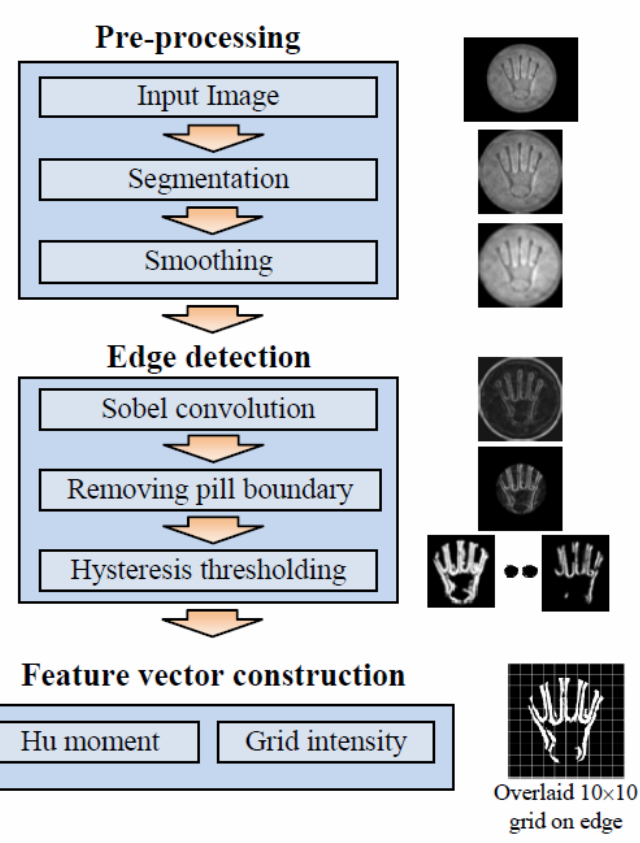

Figure 2. Schematic of feature extraction process.

\section{Multi-hysteresis thresholding}

We use a set $T=t_{1}, t_{2}, \ldots, t_{6}$ of threshold values, defined as:

$$
t_{i}=(0.1+(0.02 \times(i-1))) \times(\max -\min )+\min
$$

where $i=1, \ldots, 6$ and $\max$ and $\min$ are the maximum and minimum values of the Sobel response, respectively. For each $t_{i}, t_{i}^{\text {high }}=t_{i} \times$ high and $t_{i}^{\text {low }}=t_{i} \times$ low are also defined with high $=2.5,3,3.5$ and low $=1,2$. In the thresholding with hysteresis, all pixels that are larger than $t_{i}^{\text {high }}$ are first marked as edges. Then, any adjacent pixel of an edge whose Sobel response is larger than $t_{i}^{\text {low }}$ is also marked as an edge in the second round. With six different values of $t$, three values of high and two values of low, we can generate 36 different edge images from each Sobel response. Fig. 3 shows an example pill image and its 36 different edge images produced by the multi-hysteresis thresholding method.

\section{Feature vector construction}

We use a set of moment invariants introduced by $\mathrm{Hu}$ [11] and a grid intensity method to construct the feature vector. Given an edge image $E(x, y)$, the central moment of order $(a+b)$ is defined as

$$
\mu_{a, b}=\sum_{(x, y) \in E}(x-\bar{x})^{a}(y-\bar{y})^{b} E(x, y)
$$

where $(\bar{x}, \bar{y})$ is the center of the image. Based on the $2^{\text {nd }}$ and $3^{\text {rd }}$ order moments $((a+b)=2,3)$, a set of seven 




Figure 3. An example drug-pill image and its 36 edge images from multihysteresis thresholding.

features that are invariant to translation, rotation, and scale are obtained [11]. The grid intensity method collects the edge values from each block separated by a set of grids (Fig. 2).

\section{E. Matching}

Given a query image $(q)$ and $N$ gallery images $(g)$, all $K(=36)$ different feature vectors of the query are compared with the feature vectors of all the gallery images. The closest match of the $m^{\text {th }}$ query is selected as

$$
I D_{m}=\arg \min _{n} d\left(q_{m}^{i}, g_{n}^{j}\right)
$$

where $n=1, \ldots, N, i, j=1, \ldots, K$ and $d(.,$.$) is the dis-$ tance metric using the L2-norm. The $I D(=n)$ of the closest gallery image is assigned as the $I D$ of the query (Fig. 4). We calculate the matching accuracy using Hu moments, grid intensity and their fusion. Min-max normalization and weighted sum method is used for the fusion.

\section{EXPERIMENTAL RESULTS}

\section{A. Database}

We received 822 illicit drug pill images from the Australian Federal Police. We also downloaded 1,294 legal drug pill images from the web sites of the U.S. Drug Enforcement Administrations Office of Forensic Sciences (DEA) [3], Drug information online [5] and pharmer.org [13]. Among these 2,116 images, 602 illicit drug pill images contain at least two duplicate images belonging to the same imprint pattern (88 distinctive patterns). The remaining 1,514 images did not contain any duplicates. Image size of the pills varies from $48 \times 42$ to $2,088 \times 1,550$ (width $\times$ height) with $96 \mathrm{dpi}$ resolution.

\section{B. Matching results}

We used leave-one-out method to match each of the 602 query images that have duplicate(s) to the entire image database $(N-1=2,115)$. The Cumulative Match characteristic (CMC) curves for both single and multi-enrollment

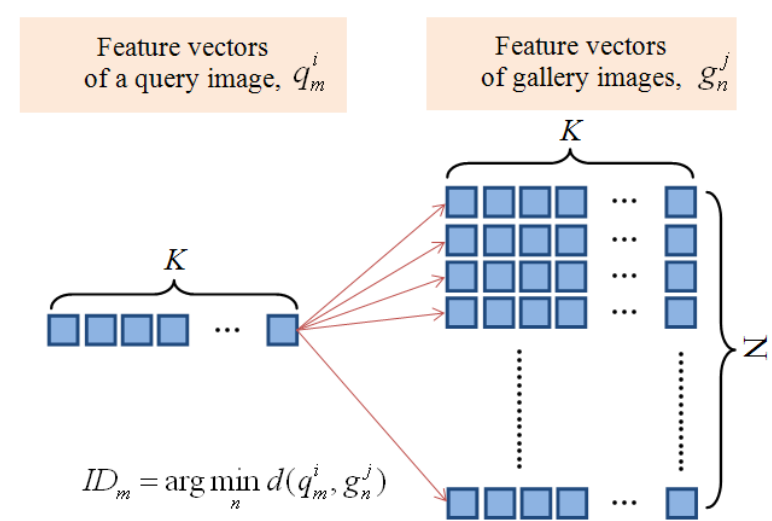

Figure 4. A schematic of the proposed matching scheme using multiple template enrollment.

methods with grid intensity and $\mathrm{Hu}$ moment based feature vectors are shown in Fig. 5. It is observed that the multienrollment method for both types of features shows much better matching accuracy due to a reduction in the intraclass variation. It is also observed that the grid intensity method performs better than the Hu moments. We believe the 7-dimensional feature vector from $\mathrm{Hu}$ moments is not sufficiently large to distinguish different patterns compared to the 100-dimensional feature vector from grid intensity. The best performance is obtained by the fusion of $\mathrm{Hu}$ moments and grid intensity. The weights are empirically chosen as .2 and .8 for $\mathrm{Hu}$ moments and grid intensity, respectively.

Matching results for ten example queries using multitemplate enrollment with grid intensity features are shown in Fig. 6 . The first four queries in Fig. 6 are correctly matched at rank-1. The last six queries are not matched correctly at rank-1, but were correctly matched at rank-2, 3, 5, 6, 4 and 4 , respectively. Failed matching examples at rank1 are due to the severe illumination changes and rotation variations. The proposed method is implemented in Matlab; the average feature extraction time based on grid intensity and $\mathrm{Hu}$ moments are 1.33 and 2.35 sec., respectively on a $2.4 \mathrm{GHz}$ CPU and 4GB memory PC. Matching time is negligible due to the simple matching scheme used here. Considering the low computation time and high matching accuracy, matching with grid intensity feature with multitemplate enrollment is the most effective method supported by our experiments.

\section{CONCLUSION}

We have proposed an automatic illicit drug pill identification method based on the shape of the imprints on the pill tablets. We have tested the proposed method on a pill image database containing a total of 2,116 images with 822 illicit drug pill images. Rank-1 (Rank-20) identification accuracy of $76.74 \%(93.02 \%)$ is obtained in the matching 




Figure 5. CMC curves for pill matching.

experiments. This method can be used to identify illegal drug pills to assist law enforcement units by providing information such as the chemical composition and their mix, manufacturer and manufacturing location for investigative purposes. The proposed illicit drug pill identification method can also be used to identify legal drug pills or tablets when the prescription information is lost.

We are improving the current system to incorporate size, shape and color of the pills in addition to the imprint. The extended method will especially help in identifying pills with missing or damaged imprints. Other representation and matching schemes are also being explored using gradient based descriptors (e.g., PHOG [14]) and subspace classifiers.

\section{ACKNOWLEDGMENT}

Authors would like to thank Dr. Mark Tahtouh of Australian Federal Police for providing drug pill images. Anil K. Jain's research was partially supported by WCU (World Class University) program through the National Research Foundation of Korea funded by the Ministry of Education, Science and Technology (R31-2008-000-10008-0).

\section{REFERENCES}

[1] National Drug Threat Assessment, National Drug Intelligence Center, U.S. Department of Justice, December 2009.

[2] J. Walters, Ed., Drug Policy Information Clearinghouse Fact Sheet, Office of National Drug Control Policy, March 2000.

[3] U.S. drug enforcement administration's office of forensic sciences. [Online]. Available: http://www.justice.gov/dea/ programs/forensicsci/microgram/index.html

[4] Code of Federal Regulations, Title 21., CFR Std.

[5] Pill identifier - drugs.com. [Online]. Available: http: //www.drugs.com/pill_identification.html

[6] European monitoring centre for drugs and drug addition. [Online]. Available: http://www.emcdda.europa.eu/index.cfm
Query

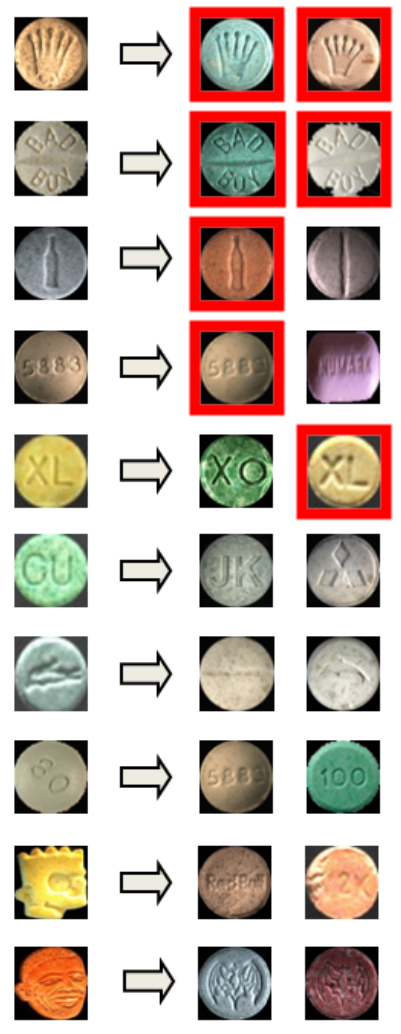

Rank-1 2
Top-6 retrievals


(



(19)
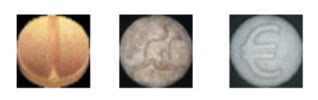

C)
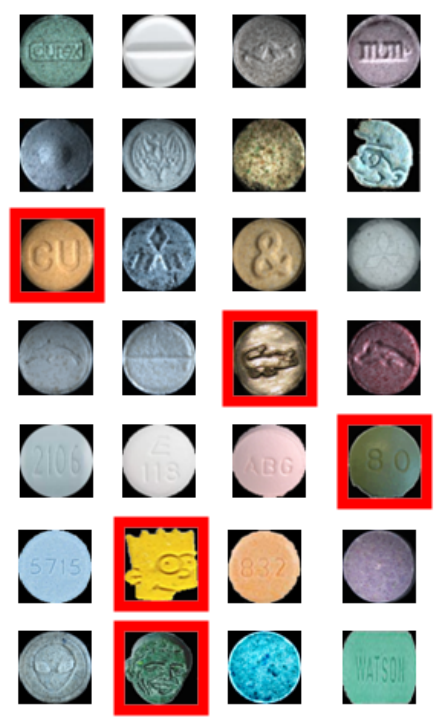

34
Figure 6. Example matching results. Correct matches in the top-6 retrieved images are enclosed with red boxes.

[7] Pill identification - webmd. [Online]. Available: http: //www.webmd.com/pill-identification/default.htm

[8] Z. Geradts, "Content-based information retrieval from forensic image databases," Ph.D. dissertation, University of Utrecht, 2002.

[9] M. Riesenhuber and T. Poggio, "Models of object recognition." Nature neuroscience Supplement, vol. 3, pp. 11991204, November 2000.

[10] J. Canny, "A computational approach to edge detection," IEEE Trans. Pattern Anal. Mach. Intell., vol. 8, no. 6, pp. 679-698, 1986.

[11] M.-K. Hu, "Visual pattern recognition by moment invariants," IEEE Transactions on Information Theory, vol. 8, no. 2, pp. 179-187, 1962.

[12] R. O. Duda and P. E. Hart, Pattern Classification and Scene Analysis. John Wiley \& Sons Inc., 1973.

[13] Image galleries - pharmer.org. [Online]. Available: http: //www.pharmer.org/images

[14] A. Bosch, A. Zisserman, and X. Munoz, "Representing shape with a spatial pyramid kernel," in Proc. ACM int'l conf. on Image and Video Retrieval (CIVR), 2007, pp. 401-408. 